\title{
Paradigm Shift: An Eco-friendly Approach to Create and Display Stereoscopic Geometric Designs
}

\author{
Lin Hsin Hsin \\ INFOTECH Research \& Consultancy \\ 75 Meyer Road \#13-0, Singapore 437901 \\ mathematicxx@gmail.com
}

\begin{abstract}
Conventionally, a 2D image can be captured by a camera, composed and painted by a 2-button mechanical mouse (a digital brush) with a digital palette. A 3D object can be modeled by 3D (translate, rotate, scale, transform) software or formulated by equation-based method. Be it 2D images or 3D objects, the result can be displayed on a 2D image on screen or print it on a given surface. The color and lighting conditions can be embedded in the software in a single or multistep process. While stereopsis is as old as the built-in capability of the human or animal (cats and apes), the desire for enabling stereopsis was first noted by Leonardo da Vinci, $15^{\text {th }}$ Century. Subsequently, various stereoscopic 3D displays was manifested in 3D imaging by David Brewster, 1807, lenticular display in 1915, 3D television by John Logie Baird, 1928, the latest being the US235 million 3D movies Avatar premiered in December 2009.
\end{abstract}

Many attempts have been made to reproduce human stereo vision deploying sophisticated software and hardware at prohibitive costs, albeit with mammoth commercial success. To experience this 3D phenomenon, a common practice to view such illusion-of-depth effects is to provide the archetype 3-D glasses to view anaglyph images, or polarized 3D glasses from the $80 \mathrm{~s}$, or LCD shutter glasses using the ellipse method and the increasing emergence of new 3-D viewing systems without special glasses. Autostereoscopic LCD displays include the lenticular lens-based Alioscopy 3D LCD display screens since mid 1990s, Sharp Actius RD3D notebook, 2004, Hitachi mobile phone in 2009, and handheld gaming device Nintendo 3DS, 2010. To date, autostereoscopy has increasingly become a method of displaying stereoscopic images without the use of special headgear or glasses by the viewer, albeit there is still the "obligatory" 3D display hardware in use. In lieu of such commercial euphoria, there is a pertinent need and hence to device a method to produce autostereoscopic effects on existing flat-panel display.

This paper offers and presents an eco-friendly and cost effective possibilities to create and view two categories of illusion-of-depth effects, viz: lenticular lens and stereoscopy instantaneously. Fundamentally, it is based on the rudiments of colors, the principal of spatial visualization and the language of spatial constructs. The approach is to define a geometric design, create a structural displacement, coupled with appropriate color interactions to resonate the perception of eyes, and hence provide the illusion-of-depth effects. As such, this conjugation demonstrates the results of an unprecedented create-and-display stereoscopic efforts of a huge repertoire of generative or non-generative simple and/or geometric designs with moderate complexities. It includes, but is not limited to 3D metallic threads, bamboo and rattan weaves, fabrics such as batik, linen, tweed and tartan. Therein, interlaced images, sequels, coleuraille (beyond grisaille and grunaille) as well as true depth-of-illusion effects can be achieved without additional hardware manufacturing, software development and operational costs. With this native access to the viewer's own display technology without the need for special configuration settings. This approach ensures high availability, no device compatibility trans-codings with on-demand performance efficiency. It illustrates the single process create-and-view direct approach based on the concept herein. More research will be conducted in the near future to achieve stereoscopy for a wider range of subject matters.

Keywords: stereoscopic images, spatial visualization, illusion-of-depth, perception of eyes, hardware assisted display, 3D geometric design, creation and display, color and geometry, linear perspective, lenticular lens, lenticular printing, grisaille, coleuraille 


\section{INTRODUCTION}

Fundamentally, a stereoscopic 3D image is based on the rudiments of colors, the principal of spatial visualization and the language of spatial constructs. In this paper, the approach to stereoscopic images is to define a geometric design, create a structural displacement, coupled with appropriate color interactions to resonate the perception of eyes, and hence provide the illusion-of-depth effects. As such, in lieu of the foray of recent studies in both hardware and software driven interests and research in multi-layer, time-multiplexed, multi-view auto/non-auto stereoscopic images and video generation; hardware assisted devices such as IRcontrolled active shutter, head-mounted display, pulsed LED-LCD projectors, glasses-based or glasses-free displays, this conjugation demonstrates the results of an unprecedented inexpensive create-and-display stereoscopic efforts of a huge repertoire of generative or nongenerative simple and/or geometric designs with moderate complexities free of visual fatigue.

\section{STEREOSCOPY}

\subsection{Definition}

Stereoscopy creates the illusion of depth from images on a two-dimensional plane based on the fact that all points in the image focus at the same plane regardless of their depth in the original scene. Basically, it is presenting two images of the same subject, each from a different position, then "informing" the brain into merging these images into one. In addition, lightings, colors different orientations and/or viewpoints can result in different illusions.

A properly constructed stereoscopic image is aligned vertically on the display screen, so onscreen binocular disparities are strictly horizontal. As and when the viewer's inter-ocular axis is also horizontal, he/she makes horizontal vergence eye movements to fuse the stereoscopic image.

\subsection{Categories of Illusions}

Presented herein are categories of illusions that can possibly be achieved in generating stereoscopic 3D geometric designs.

\subsubsection{Color and Geometry}

Traditionally, a perfect identity with an object with the most faithful resemblances of real objects described with shadowing and coloring was used to present to the mind of the observer a stereoscopic view. However, stereoscopic view does not limit itself upon the representation of real objects only. As such, the deployment of colors and geometry that constitute the arrangement in scene enable and influence the depth of view, in part or in whole (Smith \& Collar 2012). Hence it is possible to construct and present an abstract geometric composition or structure with stereoscopic 3D view. In addition, it is entirely possible to observe different constructs with different designated or prescribed viewing distance.

\subsubsection{Linear Perspective}

\section{Definition}

Linear Perspective is a representation formed by the convergence of parallel edges seen from a sufficiently distant viewpoints. The depth is conveyed primarily by the linear perspective of the angles of the horizontals whereby all horizontal structures (dis)appearing into the distance away from the image plane should project to a single central vanishing point (Weigle, 2008).

\subsubsection{Lenticular Images}

\section{Definition}

grisaille (French, from gris, gray) is a decoration in monotones especially gray designed to produce a three-dimensional effect. Hence it is possible to create an extension of grisaille, that is, coleuraille, a decoration in any and multiple colors for producing three-dimensional effect. Therein, the interlaced images, sequels, grisaille, grunsaille or coleuraille is the formation of a lenticular image, which can be used for lenticular printing or animation.

\subsubsection{Hybrid}

It is possible to create stereoscopic 3D design with any two of the categories defined herein.

\section{Existing Methodologies}

\subsection{Capturing and Generating Stereoscopic 3D Images}

Listed below are several methods known to date used for capturing and generating a stereoscopic 3D images.

i The simplest of generating a stereoscopic 3D images is a stereo rig with two cameras precisely matched and aligned, while focusing on single camera precision. Any correction of the deviations there arise can be performed either live or during post-production. This process is limited to stationary objects or the use of synthetically 
created animations (Devernay, Pujades \& Vijay 2012)

ii Depth imbued stereoscopic 3D images can also be generated by aligning two or more cameras along the same horizontal axis, point and shoot the same scene or object from different viewpoints, wherein controlling and presenting a camera footage to the left or right eye, hence the binocular parallax to induce depth sensation.

iii Scalable multi-view stereo camera array used for real-time image capture (Hill, 2004)

iv software-based method to convert $2 \mathrm{D}$ images to stereoscopic 3D images according to the monocular depth cues (Koido \& Kawai 2012).

\subsection{Viewing and Displaying Stereoscopic 3D Images}

Stereoscopic 3D images can be viewed and displayed on several commercially available 3D LCD monitors, either viewed with glasses or glassfree. Beyond which, current non-commercial 3D stereoscopic image display include:

i Multi-focal display for displaying images in focus on geometrically and radiometrically complex surfaces (Watt, MacKenzie \& Ryan 2012) .

ii Multilayer displays constructed by stacking multiple liquid crystal panels along with a pair of polarizers and a light source (Gotoda, 2012).

iii Floating image display (Chen et al 2012)

iv Seamless multi-projector blending for supporting immersive applications (Chamaret, Boisson \& Chevance 2012).

v Set up a small number of high-speed projectors and a rotating screen (Uchida \& Takaki 2012)

vi Super-multiview display device.

vii Active Shutter Glasses (a.k.a. Liquid Crystal Shutter (LCS) 3D glasses or just Shutter Glasses for time-sequential stereoscopic displays (Woods \& Helliwell 2012).

viii Eye-tracked head-mounted display (ET-HMD) system emulating classical HMD to display virtual images coupled with tracking eye gaze (Hua \& Gao 2012).

ix Specially constructed 3D display system consists of multiple basic 3D display units without a screen. The basic unit consists of a micro-projector, a lenticular lens array sheet, and a cylindrical lens deploying the light rays from a micro-projector that pass through a lenticular lens array sheet and be observed directly (Takahashi, Hirooka \& Yamada 2012).

$x$ Specially constructed glasses-free 3D display for displaying stereoscopic 3D images on a large screen using multi-projectors and an optical screen consisting of a special diffuser film with an optimally designed large condenser lens capable of using natural parallax images (Kawakita et al 2012).

xi Multi-view video and depth coding system for multiview video applications such as 3DTV.

xii View synthesis based on the concept of missing area being filled with the background parts to generate a virtual scene in a desired view point (Kim et al, 2012)

\subsection{Known Deficiencies}

Several known hardware and software deficiencies have been reported:

i Occurrence of perspective distortion when relative disparity between elements are detected (Doyen, Sacré \& Blondé 2012).

ii Misalignment correction for stereoscopic 3D camera (Pekkucuksen, Batur \& Zhang 2012).

iii Depth noise reduction, depth super-resolution for stereoscopic image synthesis.

iv Reduction of flicker at low-refresh rate (Kakeya \& Kodaira 2012)

$v$ Most of the autostereoscopic display suffer the degradation of resolution, and hence the need for enhancement of resolution, pixel density, color, and interactivity (Kwon \& Choi 2012)

vi Degrading image quality due to the increase of image size (Kawakita et al 2012)

vii Occurrence of perspective distortion due to the relative disparity between elements generating a depth not in accordance with the relative size of the presented objects (Doyen, Sacré \& Blondé 2012)

viii Despite the widespread 3D television and freeviewpoints television the field of view is still somewhat restrictive (Kim et al, 2012), (Conze, Philippe, Morin 2012)

ix Defined as the leakage of one eye's image into the image of the other eye, this inadequacy affects all commercially available stereoscopic viewing systems (Tsirlin, Allison \& Wilcox 2012) 
$x$ Overcoming the issue of heterogeneous viewing conditions in stereoscopic delivery for 3D movies in cinema (Boisson \& Chamaret 2012). Hence the necessity for displaying a perspective-correct stereoscopic views - adjustable spatio-angular display for multiple, individually tracked viewer users.

xi View-dependent geometric correction for projecting images onto geometrically complex surfaces without causing image deformations

\subsection{Stereoscopic 3D Contents}

The high costs of generating stereoscopic 3D Contents for 3-D capable consumer electronics such as 3-D cameras and TVs outweighs the availability of the devices. The Stereoscopic 3D proliferation on PCs is mired by several technical and business challenges.

Typically, consumer generated content is viewed at a close distance which makes the vergenceaccommodation conflict particularly pronounced, and hence causing discomfort and eye fatigue (Zhang, Kothandaraman \& Batur 2012). Beyond which, observing 3D content on a cinema or TV screen potentially generates visual fatigue as well.

There is even a suggestion on creating multi-layer attenuation-based designs (Lanman et al 2012)

\subsection{Visual Fatigue, Discomforts}

Apart from hardware deficiencies, one important Inherent problem of viewing stereoscopic 3D images is the apparent viewing discomfort due to cross-talk amongst stereo images

As such, visual discomfort (Kim, Kane \& Banks 2012) assessment (Hosik et al 2012) and QoE (quality of experience) (Chen et al 2012) to viewers need to be addressed and conducted. In particular, hardware dependent equipments for enhancement of perceived depth of multi parallel, overlapping, transparent, stereoscopic surfaces (Aida, Shimono \& Tam 2012) as well as reducing eye strains, headaches (Atanassov et al 2012) and visual fatigues from vertical and torsional disparities (Tyler et al, 2012), eye movements (Vienne, Blondé \& Doyen 2012) ensue the need to improve current deficiencies.

\section{$4 \quad$ New Method}

While waiting for the possibility of manufacturing low-power and low-cost 3D sensing and display devices, the availability of the current 3D hardware has so far outpaced the production of 3D content. This paper offers and presents the results of an unprecedented eco-friendly and cost effective possibilities to create-and-view three categories of illusion-of-depth and highly scalable stereoscopic 3D images instantaneously: a huge repertoire of generative or non-generative simple and/or geometric designs with moderate complexities with the following attributes

i Images to be generated, viewed and displayed without installing extra hardware

ii As stereoscopic 3D content should be captured for a specific viewing geometry in order to provide a satisfactory 3D experience, the viewing distance for these algorithm-based images can be calculated and adjusted accordingly.

iii The scalability of these stereoscopic 3D images can be purposed for billboard size screen, projector, any PC LCD or LED screen, laptop, TV and mobile devices that include tablet, smart phone without supporting stereoscopic display, and hence eliminating the need for retargeting the content.

iv As it does not require any head mount display and/or glasses, viewing these stereoscopic 3D images does not cause head and eyes discomforts and fatigues.

\subsection{The Results}

The result of this algorithm-based creation and display of stereoscopic 3D images presented here is in accordance to the specified categories described in paragraph 2 . These images are highly scalable, exhibit the same Quality of Experience regardless of size, maintaining the minimal file size at creation time, and it is without additional storage burden during viewing and display.

The indicated viewing distance here is suitable for a desktop PC screen or laptop. It can be adjusted accordingly when viewing on different display devices. All works are created on a Linux platform.

\section{Category 1 Color and Geometry}

Figure 1: Look Into by Lin Hsin Hsin, 2010 single-layer, multi-view

Viewing angle:parallel to xy plane or $30^{\circ}$ to $x z$ plane Viewing distance: $38 \mathrm{~cm}$

Figure 2: Watercolor by Lin Hsin Hsin, 2010 single-layer, multi-view

Viewing angle:parallel to xy plane or $30^{\circ}$ to $x z$ plane Viewing distance: $38 \mathrm{~cm}$

\section{Category 2 Linear Perspective}

Figure 3: Look Inside by Lin Hsin Hsin, 2010 multi-layer, multi-view

Viewing angle:parallel to xy plane. Viewing distance:38cm 
Figure 4: Look Away by Lin Hsin Hsin, 2010 multi-layer, multi-view

Viewing angle:parallel to xy plane. Viewing distance: $38 \mathrm{~cm}$

Figure 5: Multifold by Lin Hsin Hsin, 2011 single-layer, multi-view

Viewing angle:parallel to $x y, x z$ or $y z$ plane. Viewing distance: $38 \mathrm{~cm}$

\section{Category 3 Lenticular Images}

Figure 6: In Frost We Crust by Lin Hsin Hsin, 2010 multi-layer, multi-view

Viewing angle:parallel to xy plane. Viewing distance: $38 \mathrm{~cm}$

Figure 7: Bamboo Green by Lin Hsin Hsin, 2010 multi-layer, multi-view

Viewing angle:parallel to xy plane. Viewing distance: $38 \mathrm{~cm}$

Figure 8: 4 Seasons by Lin Hsin Hsin, 2010 multi-layer, multi-view

Viewing angle:parallel to $x y, x z$ or $y z$ plane. Viewing distance: $38 \mathrm{~cm}$

\section{Category 4 Hybrid}

These two examples illustrate the combination of Category 1 and 2

Figure 9: Crystals by Lin Hsin Hsin, 2011 multi-layer, multi-view

Viewing angle:parallel to xy plane. Viewing distance: $38 \mathrm{~cm}$

Figure 10: Perspectives by Lin Hsin Hsin, 2010 multi-layer, multi-view

Viewing angle:parallel to $x y, x z$, or $y z$ plane. Viewing distance: $38 \mathrm{~cm}$

\section{$5 \quad$ Conclusions}

As dissemination of $3 \mathrm{D}$ technologies at cinemas and in consumer products trend to become a lifestyle, This inexpensive method described herein allows a viewer native access to the viewer's own display technology without the need for special configuration settings and additional hardware without visual and eyes fatigues. This approach ensures high availability, no device compatibility trans-codings with on demand performance efficiency. It illustrates the single process createand-view direct approach based on the concept herein. More research will be conducted in the near future to achieve stereoscopy for a wider range of subject matters that merit artistic potentials.

\section{References}

Weigle, C. A Comparison of the Perceptual Benefits of Linear Perspective and PhysicallyBased Illumination for Display of Dense 3D Streamtubes, Visualization and Computer
Graphics, Nov.-Dec. 2008, IEEE Transactions on Vol 14, Issue: 6, pp. 1723 - 1730

Smith Michael D. and Collar Bradley T., Perception of size and shape in stereoscopic 3D imagery, Stereoscopic Displays and Applications XXIII, San Francisco, USA, 23-25 January 2012, Proceedings of SPIE Vol 8288. http://dx.doi.org/10.1117/12.912205

\section{Capturing}

Frédéric Devernay, Sergi Pujades, and Vijay Ch. A.V., Focus mismatch detection in stereoscopic content, Stereoscopic Displays and Applications XXIII, San Francisco, USA, 23-25 January 2012, Proceedings of SPIE Vol 8288 http://dx.doi.org/10.1117/12.906209

Hill Samuel L., Scalable Multi-view Stereo Camera Array for Real World Real-Time Image Capture and Three-Dimensional Displays, 2004

Koido Yoshihisa and Kawai Takashi, Partial 2D to S3D conversion and the cognitive characteristics., Stereoscopic Displays and Applications XXIII, San Francisco, USA, 23-25 January 2012, Proceedings of SPIE Vol 8288. http://dx.doi.org/10.1117/12.910662

Chen Chang-Ying, Tseng Kun-Lung, Wang ChyLin, and Tsai Chao-Hsu, Floating image device with autostereoscopic display and viewer-tracking technology, Stereoscopic Displays and Applications XXIII, San Francisco, USA, 23-25 January 2012, Proceedings of SPIE Vol 8288 http://dx.doi.org/10.1117/12.912042

Doyen D, Sacré J.-J., and Blondé L, Correlation between a perspective distortion in a S3D content and the visual discomfort perceived, Stereoscopic Displays and Applications XXIII, San Francisco, USA, 23-25 January 2012, Proceedings of SPIE Vol 8288. http://dx.doi.org/10.1117/12.906735

Viewing and Displaying

Watt Simon J., MacKenzie Kevin J., and Ryan Louise, Real-world stereoscopic performance in multiple-focal-plane displays: How far apart should the image planes be? Stereoscopic Displays and Applications XXIII, San Francisco, USA, 23-25 January 2012, Proceedings of SPIE Vol 8288. http://dx.doi.org/10.1117/12.908883

Gotoda, Hironobu, Implementation and analysis of an autostereoscopic display using multiple liquid crystal layers, Stereoscopic Displays and Applications XXIII, San Francisco, USA, 23-25 January 2012, Proceedings of SPIE Vol 8288. http://dx.doi.org/10.1117/12.909881 
Chamaret C., Boisson G., and Chevance C., Video retargeting for stereoscopic content under 3D viewing constraints, Stereoscopic Displays and Applications XXIII, San Francisco, USA, 23-25 January 2012, Proceedings of SPIE Vol 8288. http://dx.doi.org/10.1117/12.908445

Uchida Shigeki and Takaki Yasuhiro, 360-degree three-dimensional table-screen display using small array of high-speed projectors, Stereoscopic Displays and Applications XXIII, San Francisco, USA, 23-25 January 2012, Proceedings of SPIE Vol 8288. http://dx.doi.org/10.1117/12.909603

Woods Andrew J. and Helliwell Jesse, Investigating the cross-compatibility of IR-controlled active shutter glasses, Stereoscopic Displays and Applications XXIII, San Francisco, USA, 23-25 January 2012, Proceedings of SPIE Vol 8288. http://dx.doi.org/10.1117/12.912061

Hua Hong and Gao Chunyu, A compact eyetracked optical see-through head-mounted display, Stereoscopic Displays and Applications XXIII, San Francisco, USA, 23-25 January 2012, Proceedings of SPIE Vol 8288. http://dx.doi.org/10.1117/12.909523

Takahashi Hideya, Hirooka Kenta, and Yamada Kenji, Wide-viewing angle three-dimensional display based on the ray reconstruction method using multiple micro-projectors, Stereoscopic Displays and Applications XXIII, San Francisco, USA, 23-25 January 2012, Proceedings of SPIE Vol 8288. http://dx.doi.org/10.1117/12.907254

Kawakita M., Iwasawa S., Sakai M., Haino Y., Sato M., and Inoue N., 3D image quality of 200 -inch glasses-free 3D display system, Stereoscopic Displays and Applications XXIII, San Francisco, USA, 23-25 January 2012, Proceedings of SPIE Vol 8288. http://dx.doi.org/10.1117/12.912274

Kim Seona, Lee Kyong Joon, Kim Chan, Yun II Dong, and Lee Sang Uk, A layered inpainting method for virtual view synthesis, Stereoscopic Displays and Applications XXIII, San Francisco, USA, 23-25 January 2012, Proceedings of SPIE Vol 8288. http://dx.doi.org/10.1117/12.910412

\section{Deficiencies}

Doyen D., Sacré J.-J., and Blondé L., Correlation between a perspective distortion in a S3D content and the visual discomfort perceived, Stereoscopic Displays and Applications XXIII, San Francisco, USA, 23-25 January 2012, Proceedings of SPIE Vol 8288. http://dx.doi.org/10.1117/12.906735

Pekkucuksen Ibrahim E., Batur Aziz Umit, and Zhang Buyue, A real-time misalignment correction algorithm for stereoscopic 3D cameras, Stereoscopic Displays and Applications XXIII, San Francisco, USA, 23-25 January 2012,
Proceedings of SPIE Vol 8288. http://dx.doi.org/10.1117/12.90690

Kakeya Hideki and Kodaira Hiroaki, Full color stereoscopy with little flicker at low refresh rate by time-division multiplexing anaglyph, Stereoscopic Displays and Applications XXIII, San Francisco, USA, 23-25 January 2012, Proceedings of SPIE Vol 8288. http://dx.doi.org/10.1117/12.907572

Kwon Hyunkyung and Choi Hee-Jin, A timesequential multiview autostereoscopic display without resolution loss using a multi-directional backlight unit and an LCD panel, Stereoscopic Displays and Applications XXIII, San Francisco, USA, 23-25 January 2012, Proceedings of SPIE Vol 8288. http://dx.doi.org/10.1117/12.907793

Kawakita M., Iwasawa S., Sakai M., Haino Y., Sato M., and Inoue N., 3D image quality of 200 -inch glasses-free 3D display system, Stereoscopic Displays and Applications XXIII, San Francisco, USA, 23-25 January 2012, Proceedings of SPIE Vol 8288. http://dx.doi.org/10.1117/12.912274

Kim Min Chang, Hwang Yong Seok, Hong SukPyo, and Kim Eun Soo, Design of extended viewing zone at autostereoscopic 3D display based on diffusing optical element, Stereoscopic Displays and Applications XXIII, San Francisco, USA, 23-25 January 2012, Proceedings of SPIE Vol 8288. http://dx.doi.org/10.1117/12.912230

Conze Pierre-Henri, Philippe Robert, and Morin Luce, Objective view synthesis quality assessment, Stereoscopic Displays and Applications XXIII, San Francisco, USA, 23-25 January 2012, Proceedings of SPIE Vol 8288. http://dx.doi.org/10.1117/12.908762

Tsirlin Inna, Allison Robert S., and Wilcox Laurie M., Crosstalk reduces the amount of depth seen in 3D images of natural scenes, Stereoscopic Displays and Applications XXIII, San Francisco, USA, 23-25 January 2012, Proceedings of SPIE Vol 8288. http://dx.doi.org/10.1117/12.906751

Boisson Guillaume and Chamaret Christel, Disparity profiles in 3DV applications: overcoming the issue of heterogeneous viewing conditions in stereoscopic delivery, Stereoscopic Displays and Applications XXIII, San Francisco, USA, 23-25 January 2012, Proceedings of SPIE Vol 8288. http://dx.doi.org/10.1117/12.904948

Contents

Zhang Buyue, Kothandaraman Sreenivas, and Batur Aziz Umit, Auto convergence for stereoscopic 3D cameras, Stereoscopic Displays and Applications XXIII, San Francisco, USA, 23-25 January 2012, Proceedings of SPIE Vol 8288. http://dx.doi.org/10.1117/12.906768 
Lanman Douglas, Wetzstein Gordon, Hirsch Matthew, Heidrich Wolfgang, and Raskar Ramesh, Beyond parallax barriers: applying formal optimization methods to multilayer automultiscopic displays, Stereoscopic Displays and Applications XXIII, San Francisco, USA, 23-25 January 2012, Proceedings of SPIE Vol 8288. http://dx.doi.org/10.1117/12.907146

Visual Fatigues

Kim Joohwan, Kane David, and Banks Martin S., Visual discomfort and the temporal properties of the vergence-accommodation conflict, Stereoscopic Displays and Applications XXIII, San Francisco, USA, 23-25 January 2012, Proceedings of SPIE Vol 8288. http://dx.doi.org/10.1117/12.912223

Hosik Sohn, Yong Ju Jung, Lee Seong-il, Hyun Wook Park, and Yong Man Ro, Investigation of object thickness for visual discomfort prediction in stereoscopic images, Stereoscopic Displays and Applications XXIII, San Francisco, USA, 23-25 January 2012, Proceedings of SPIE Vol 8288. http://dx.doi.org/10.1117/12.908418

Atanassov Kalin, Ramachandra Vikas, Nash James, and Goma Sergio R., Unassisted 3D camera calibration, Stereoscopic Displays and Applications XXIII, San Francisco, USA, 23-25
January 2012, Proceedings of SPIE Vol 8288. http://dx.doi.org/10.1117/12.909616

Chen Wei, Fournier Jérôme, Barkowsky Marcus, and Le Callet Patrick, Quality of experience model for 3DTV, Stereoscopic Displays and Applications XXIII, San Francisco, USA, 23-25 January 2012, Proceedings of SPIE Vol 8288. http://dx.doi.org/10.1117/12.907873

Aida Saori, Shimono Koichi, Tam Wa James, Perceived depth of multi parallel, overlapping, transparent, stereoscopic surfaces, Stereoscopic Displays and Applications XXIII, San Francisco, USA, 23-25 January 2012, Proceedings of SPIE Vol 8291.

Tyler Christopher W., Likova Lora T., Atanassov Kalin, Ramachandra Vikas, Goma Sergio, Measuring 3D discomfort from vertical and torsional disparities in natural images, Stereoscopic Displays and Applications XXIII, San Francisco, USA, 23-25 January 2012, Proceedings of SPIE Vol 8291.

Vienne Cyril, Blondé Laurent, and Doyen Didier, Visual fatigue versus eye-movements, Stereoscopic Displays and Applications XXIII, San Francisco, USA, 23-25 January 2012, Proceedings of SPIE Vol 8288. http://dx.doi.org/10.1117/12.906994 